\title{
Deep level transient spectroscopy of anisotropic semiconductor GaTe
}

\author{
D PAL, S PAL and D N BOSE \\ Semiconductor Division, Materials Science Centre, Indian Institute of Technology, \\ Kharagpur 721 302, India \\ MS received 31 January 1994; revised 18 June 1994
}

\begin{abstract}
Deep level transient spectroscopy (DLTS) was carried out on single crystals of the layered chalcogenide p-GaTe using Schottky barriers parallel and perpendicular to the layer planes to study the possible anisotropy of the defect levels. Deep levels with the same energies $(0.28 \mathrm{eV}$ and $0.42-0.45 \mathrm{eV})$ have been found in both directions with concentrations ranging from $10^{13} \mathrm{~cm}^{-3}$ to $10^{14} \mathrm{~cm}^{-3}$ and capture cross-sections from $10^{-15} \mathrm{~cm}^{2}$ to $10^{-17} \mathrm{~cm}^{2}$. The difference in the spectra obtained from the two planes and the possible reason for the deep level energies being independent of crystal orientation are discussed.
\end{abstract}

Keywords. GaTe; deep levels; anisotropy.

\section{Introduction}

Layered structure semiconducting compounds have received much attention in recent years. GaTe belongs to the family of layered chalcogenides which includes GaSe and InSe. It has monoclinic structure with the space group $\mathrm{C}_{2}^{2}$, the unit cell being made of two layers $\mathrm{Te}-\mathrm{Ga}-\mathrm{Ga}-\mathrm{Te}$. These semiconductors have band gaps of $2.05 \mathrm{eV}(\mathrm{GaSe})$, $1.80 \mathrm{eV}(\mathrm{InSe})$ and $1.66 \mathrm{eV}$ (GaTe) respectively. Deep level transient spectroscopy (DLTS) was previously reported on p-GaSe (Shigetomi et al 1991) and n-InSe (Mari et al 1991) only along one orientation, i.e. with Schottky barriers placed on the easy cleavage planes. It was thus considered important to examine defect levels in two orthogonal directions in such anisotropic compounds in which the dielectric constants and effective masses are highly orientation-dependent. In this paper results of DLTS measurements on undoped p-type single-crystal GaTe are reported for the first time.

A schematic diagram of the crystal structure of GaTe with the Schottky contacts used in the two cases is shown in figure 1 ( $a$ and $b$ ) for clarity. For the calculation of barrier height $\left(\phi_{\mathrm{b}}\right)$, capture cross-section $(\sigma)$, etc. corresponding to electrical contacts made perpendicular to the layer planes (figure 1a), the parallel components of transport parameters such as $\varepsilon, m^{*}$, etc. are required. This is because for such a geometry the transport of carriers occurs in a direction parallel to the layer planes. Hence we have denoted these components with the suffix $\|$. For contacts made parallel to the layer planes (figure $1 \mathrm{~b}$ ) transport occurs perpendicular to the planes and hence parameters are denoted with the suffix $\perp$.

\section{Experimental}

Single crystals of $\mathrm{GaTe}$ (m.p. $824^{\circ} \mathrm{C}$ ) approximately $1 \mathrm{~cm}$ dia and $2.5 \mathrm{~cm}$ length were grown by the conventional Bridgman technique at very slow growth rates from stoichiometric melts. A photograph of a crystal placed on a cm scale is shown in figure 2 . These crystals have shiny mica-like appearance and cleave easily parallel to 


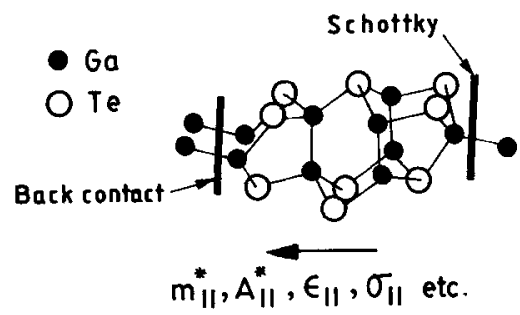

(a)

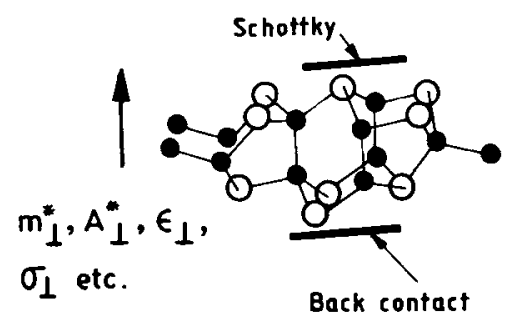

(b)

Figure 1. Crystal structure of GaTe with Schottky contacts (a) perpendicular and (b) parallel to layer planes. $m^{*}, A^{*}, \varepsilon$, etc. are explained in the text.

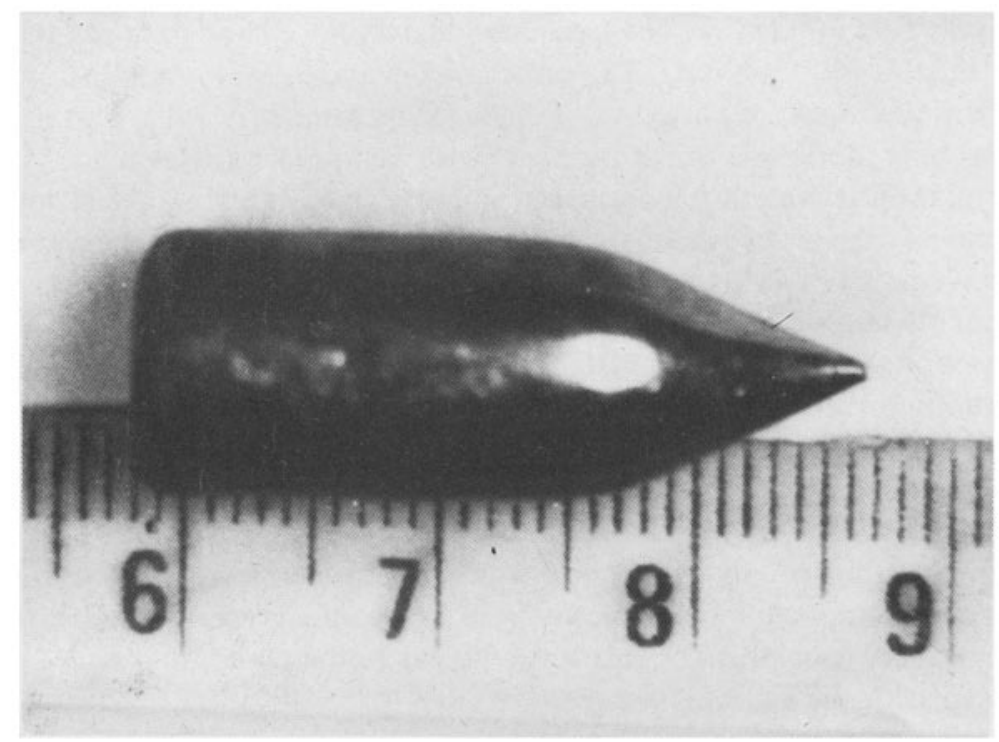

Figure 2. Photograph of the GaTe ingot.

the layer planes due to weak van der Waals bonding. Laue back-reflection studies confirmed the single-crystal structure of the material and showed the growth direction to be (001). Figure 3 shows the electron diffraction pattern of GaTe showing the sample existing as a single phase material having a monoclinic structure with lattice 


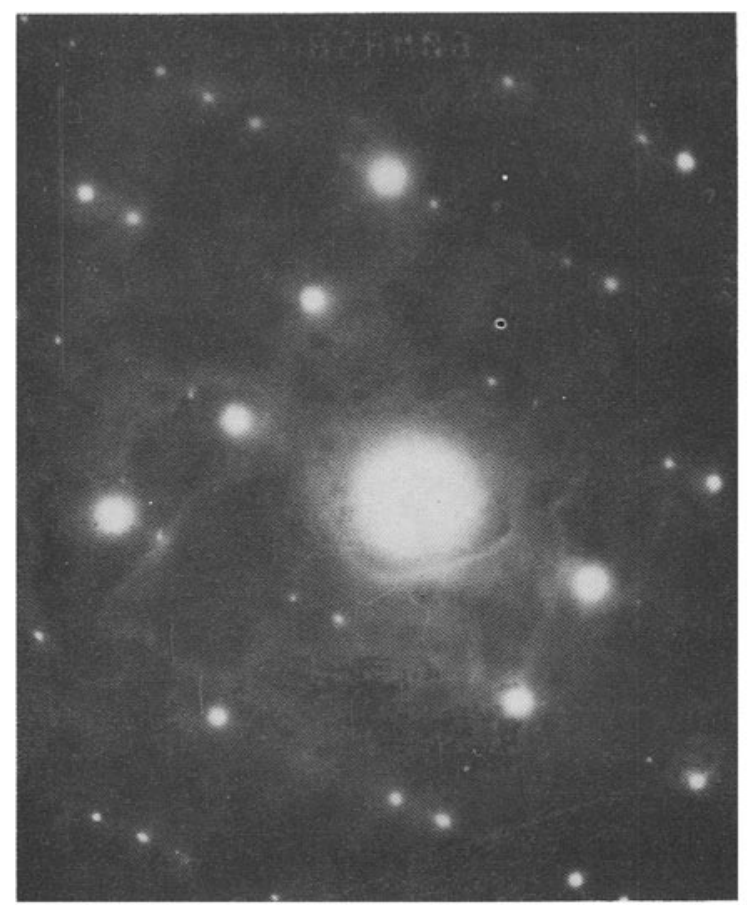

Figure 3. Electron diffraction pattern of GaTe.

Table 1. Stoichiometry of GaTe.

\begin{tabular}{lcc}
\hline & \multicolumn{2}{c}{ Composition } \\
\cline { 2 - 3 } Analysis & Ga (at. $\%)$ & Te (at. \%) \\
\hline EDAX & $49 \cdot 2-49 \cdot 3$ & $50 \cdot 7-50 \cdot 8$ \\
XPS & $49 \cdot 1-49 \cdot 2$ & $50 \cdot 8-50 \cdot 9$ \\
\hline
\end{tabular}

parameters $a=17.36 \AA, b=4.07 \AA$ and $c=10.56 \AA$. This also reveals the two-fold symmetry characteristic of a monoclinic structure.

The chemical composition of GaTe was determined by energy dispersive X-ray analysis (EDAX). The atomic percentage has been estimated from the intensity ratio of the elements. Elemental $\mathrm{Ga}$ and $\mathrm{Te}(6 \mathrm{~N}$ purity, MCP, UK) were used as standards. The chemical composition of the ingots was further checked by XPS (ESCA) analysis. Measurements were also carried out for elemental $\mathrm{Ga}$ and Te. The composition was calculated from the peak area, measured under identical conditions with Ar-ion etching. The results agreed with the values obtained from EDAX (table 1), the slight discrepancy being due to lower accuracy $(5-10 \%)$ of XPS measurements. Optical absorption measurements showed a direct gap of $1.66 \mathrm{eV}$ at $300 \mathrm{~K}$.

A detailed study of electrical conductivity and Hall mobility (to be reported) was carried out in two orthogonal directions. These experiments showed band-type conduction occurring between $80 \mathrm{~K}$ and $300 \mathrm{~K}$ with the hole mobility decreasing as 
$T^{-n}$ where $n=2.05$ and 3.30 for directions parallel and perpendicular to the layer planes respectively. The activation energies were found to be $E_{a \|}=0.023 \mathrm{eV}$ and $E_{a \perp}=0.04 \mathrm{eV}$ in two directions. The corresponding dielectric constants determined at $1 \mathrm{MHz}$ were $\varepsilon_{\|}=7 \cdot 7$ and $\varepsilon_{1}=5 \cdot 0$ respectively.

DLTS measurements were carried out on $0.5 \mathrm{~mm}^{2}$ Schottky diodes made by evaporating $\mathrm{Al}$ on a freshly cleaved face of the samples $\left(4 \times 3 \times 0.4 \mathrm{~mm}^{3}\right)$. Ohmic contacts on the back surfaces were made by evaporating In and annealing at $175^{\circ} \mathrm{C}$ for $4 \mathrm{~min}$ in argon atmosphere. For measurements perpendicular to the layer planes, samples $4 \times 3 \times 1 \mathrm{~mm}^{3}$ in size after cutting and polishing had Schottky diodes $\left(0.4 \mathrm{~mm}^{2}\right)$ prepared as before.

DLTS spectra were obtained between $80 \mathrm{~K}$ and $330 \mathrm{~K}$ using a conventional Boxcar system fabricated for this work with a reverse bias of $-1 \mathrm{~V}$ and filling pulses at $0 \mathrm{~V}$. The filling pulse width was $250 \mathrm{msec}$ to ensure saturation of the deep levels.

\section{Results}

For Schottky diodes the relation between $I$ and $V$ can be expressed by the equation

$$
I=A A^{*} T^{2} \exp \left\{-q\left(\phi_{\mathrm{b}}-V / n\right) / k T\right\},
$$

where $A, A^{*}, \phi_{\mathrm{b}}, n$ are area of the Schottky contact, effective Richardson constant, barrier height and ideality factor respectively. Here

and

$$
A_{\|}^{*}=120 m_{\|}^{*} / m_{0} \mathrm{~A} \mathrm{~cm}^{-2} \mathrm{~K}^{-2},
$$

$$
A_{\perp}^{*}=120 m_{\perp}^{*} / m_{0} \mathrm{~A} \mathrm{~cm}^{-2} \mathrm{~K}^{-2} \text {. }
$$

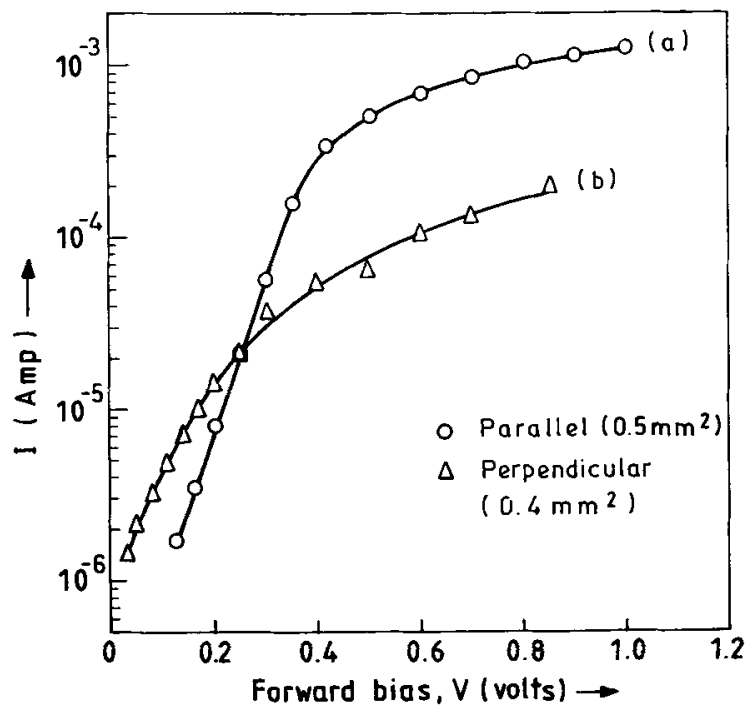

Figure 4. I vs $V$ plot of $\mathrm{Al} / \mathrm{p}-\mathrm{GaTe}$ Schottky diodes (a) parallel $\left(0.5 \mathrm{~mm}^{2}\right)$ and (b) per. pendicular $\left(0.4 \mathrm{~mm}^{2}\right)$ to the layer planes. 
Table 2. Anisotropy of transport parameters and Schottky diodes on p-GaTe.

\begin{tabular}{lccccc}
\hline & $\begin{array}{c}\rho_{300 \mathrm{~K}} \\
(\mathrm{ohm} \mathrm{cm})\end{array}$ & $\begin{array}{c}\mu_{300 \mathrm{~K}} \\
\left(\mathrm{~cm}^{2} \mathrm{~V}^{-1} \mathrm{sec}^{-1}\right)\end{array}$ & $\begin{array}{c}N_{\mathrm{A}} \\
\left(\mathrm{cm}^{-3}\right)\end{array}$ & $\begin{array}{c}\phi_{\mathrm{b}} \\
(\mathrm{eV})\end{array}$ & $n$ \\
\hline $\left.\begin{array}{l}\text { Parallel to } \\
\text { layer }\end{array}\right\}$ & $50 \pm 5.0$ & $30 \pm 2.0$ & $5.0 \times 10^{15}$ & 0.69 & 1.5 \\
$\begin{array}{l}\text { Perpendicular } \\
\text { to layer }\end{array}$ & $4.3 \times 10^{2}$ & $10 \pm 2.0$ & $3.7 \times 10^{15}$ & 0.61 & 2.5 \\
\hline
\end{tabular}

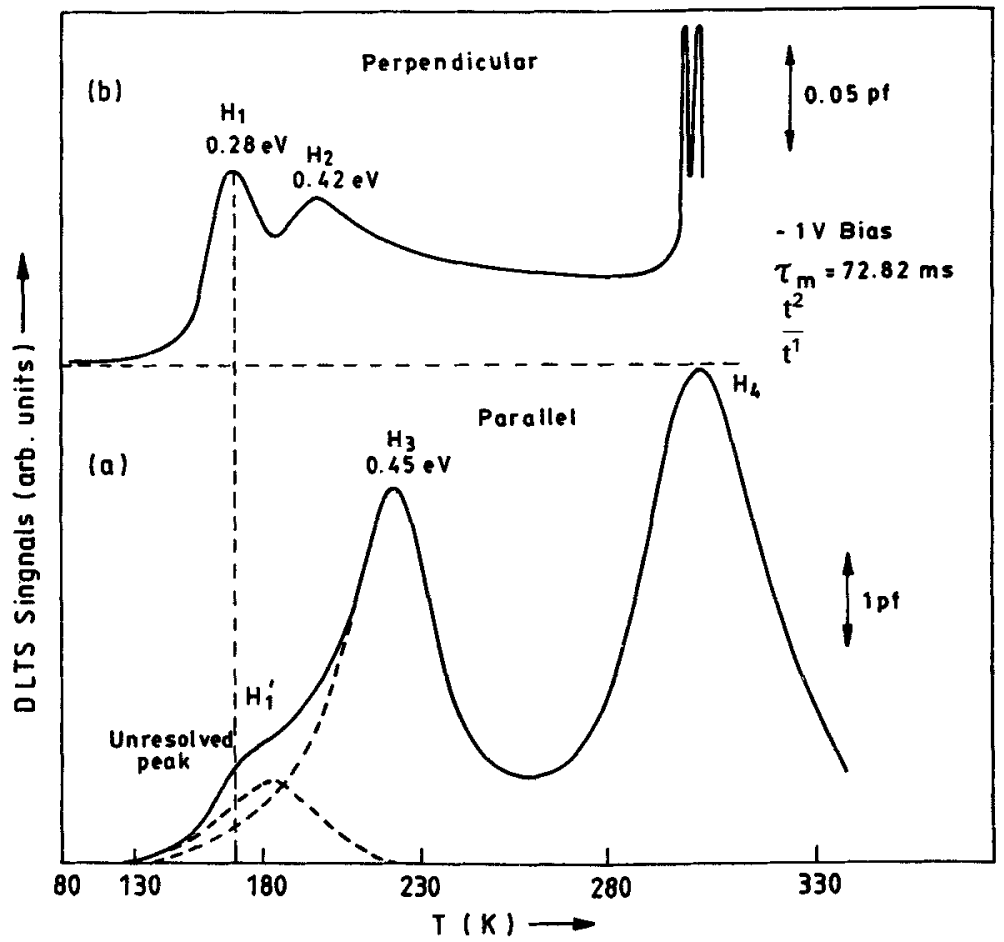

Figure 5. Typical DLTS spectra of the Schottky diodes (a) parallel and (b) perpendicular to the layer planes.

Since for p-type GaTe $m_{\|}^{*}=0.47 m_{0}, m_{\perp}^{*}=m_{0}, A_{\|}^{*}$ and $A_{\perp}^{*}$ are $56.40 \mathrm{Acm}^{-2} \mathrm{~K}^{-2}$ and $120 \mathrm{~A} \mathrm{~cm}^{-2} \mathrm{~K}^{-2}$ respectively. The plots of forward current $I$ vs $V$ of the Schottky diodes parallel and perpendicular to layer planes are shown in figure 4 (a and b). The values of $\phi_{\mathrm{b}}$ and $n$ obtained from the forward $I-V$ characteristics with other parameters of GaTe are shown in table 2. While the value of the ideality factor was 1.5 for the Schottky contact deposited on the layer plane, in the perpendicular direction the value of $n$ was 2.5 due to high density of surface dangling bonds and possible presence of stacking faults.

The DLTS spectrum for Schottky barrier parallel to the layer planes is shown in figure 5a. Two well-defined peaks $\mathbf{H}_{3}$ and $\mathbf{H}_{4}$ together with an unresolved peak $\left(\mathrm{H}_{1}^{\prime}\right)$ were observed. For $\mathrm{H}_{3}$ a thermal activation energy of $0.45 \mathrm{eV}$ was calculated from the least-square fit of the Arrhenius plot shown in figure 6. An anomalously high 
Table 3. Summary of defect parameters determined from DLTS measurements.

\begin{tabular}{lcccc}
\hline Schottky & Trap & $E_{\mathrm{T}}(\mathrm{eV})$ & $\sigma_{\infty}\left(\mathrm{cm}^{2}\right)$ & $N_{\mathrm{t}}\left(\mathrm{cm}^{-3}\right)$ \\
\hline $\left.\begin{array}{l}\text { Perpendicular } \\
\text { to layer }\end{array}\right\}$ & $\mathrm{H}_{1}$ & 0.28 & $1.0 \times 10^{-17}$ & $2.5 \times 10^{13}$ \\
$\left.\begin{array}{l}\text { Parallel } \\
\text { to layer }\end{array}\right\}$ & $\mathrm{H}_{2}$ & 0.42 & $1.7 \times 10^{-15}$ & $1.7 \times 10^{13}$ \\
& $\mathrm{H}_{1}^{\prime}$ & - & - & $1.4 \times 10^{14}$ \\
& $\mathrm{H}_{3}$ & 0.45 & $5.0 \times 10^{-15}$ & $3.6 \times 10^{14}$ \\
\hline
\end{tabular}

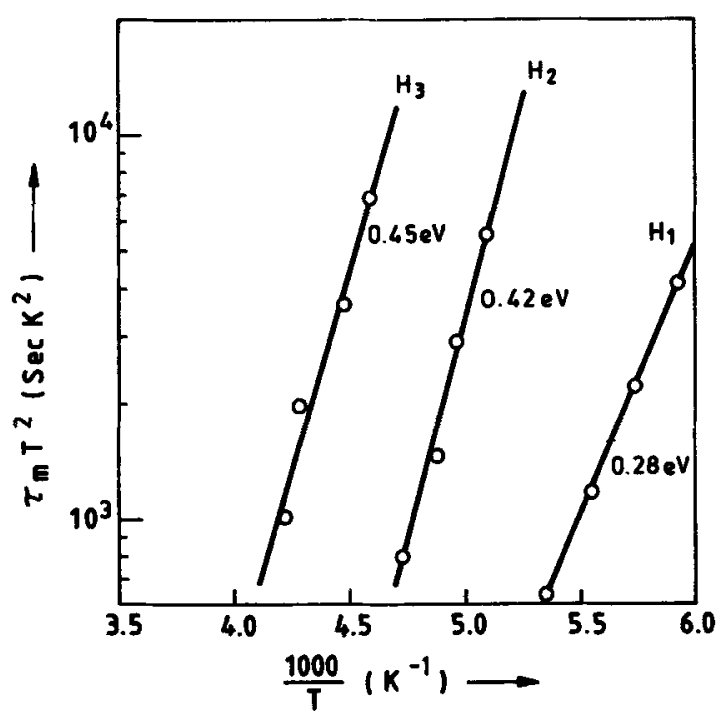

Figure 6. Arrhenius plots of thermal emission rates of levels $\mathbf{H}_{1}, \mathbf{H}_{2}, \mathbf{H}_{3}$.

capture cross-section $\left(10^{-8} \mathrm{~cm}^{2}\right)$ and high activation energy $(1 \cdot 2 \mathrm{eV})$ for $\mathrm{H}_{4}$ were obtained from the Arrhenius plot (not shown). The peak height of the DLTS signal corresponding to this trap also increased towards higher temperatures which revealed the nonexponential behaviour of the transient. This may be due to highly nonuniform distribution of traps $\left(\mathrm{H}_{4}\right)$ (Martin et al 1986) or the presence of several centres with similar energies (Telia et al 1991). Hence no activation energy could be attributed to this level.

The DLTS spectrum of sample for the Schottky barrier perpendicular to layer plane is shown in figure $5 \mathrm{~b}$. Two well-defined peaks $\mathrm{H}_{1}$ and $\mathrm{H}_{2}$ were again observed with a shoulder beyond $\mathrm{H}_{2}$ which may be due to interface states. The thermal activation energies of $\mathrm{H}_{1}$ and $\mathrm{H}_{2}$ were found to be $0.28 \mathrm{eV}$ and $0.42 \mathrm{eV}$ respectively from the Arrhenius plots (figure 6). It is clear from the DLTS spectra that the unresolved peak $\left(\mathrm{H}_{1}^{\prime}\right)$ of figure $5 \mathrm{a}$ corresponds to the deep trap peak $\mathrm{H}_{1}(0.28 \mathrm{eV})$. Its concentration was estimated by resolving the observed signal as shown in figure 5a. The trap parameters corresponding to $\mathrm{H}_{4}$ could not be determined in the perpendicular plane due to sharp increase and large fluctuations of the DLTS signals. Thermal activation energies $\left(E_{\mathrm{T}}\right)$, trap concentrations $\left(N_{\mathrm{t}}\right)$ and capture cross-sections $\left(\sigma_{\infty}\right)$ at high temperatures of $\mathrm{H}_{1}, \mathrm{H}_{2}$ and $\mathrm{H}_{3}$ are given in table 3 . 


\section{Discussion}

Deep levels are characterized by their energy levels, concentrations and capture cross-sections. While identical energies have been obtained in the case of $\mathrm{H}_{2}$ and $\mathrm{H}_{3}$ levels, possible reasons for obtaining different concentrations and cross-sections are discussed below.

The trap concentration was measured from the peak height of the DLTS signal. The concentrations of the deep levels $\mathrm{H}_{1}^{\prime}$ and $\mathrm{H}_{3}$ were higher than that of the levels $\mathrm{H}_{1}^{\prime}$ and $\mathrm{H}_{2}$ respectively. Thus it is seen that the concentration of the levels parallel to the layer plane was much higher than that perpendicular to the layer plane.

It was observed that the change in capacitance of the diode perpendicular to the plane at $200 \mathrm{~K}$ from $0 \mathrm{~V}$ to $1 \mathrm{~V}$ reverse bias was much less than for the Schottky diode parallel to the layer plane. This is due to high density of interface states present perpendicular to the layer plane, also observed from DLTS. Thus, the swing of the Fermi level between the filling pulse and the application of reverse bias was much less than that for the Schottky contact made parallel to the layer planes. Consequently the number of trapped centres which lies above the Fermi level was much less during reverse bias in the perpendicular direction. Therefore the measured hole emission was much lower in this direction than in the parallel direction resulting in the observation of a much lower concentration of the centres in the perpendicular plane.

The capture radius is defined as

$$
r_{\mathrm{c}}=e^{2} / 2 \varepsilon k T,
$$

for a Coulomb attractive potential $V(r)=-e^{2} / \varepsilon r$. Thus the capture cross-section

$$
\sigma \equiv \pi e^{4} / 4 \varepsilon^{2} k^{2} T^{2}
$$

(Bräunlich et al 1979). In this experiment capture cross-sections $\sigma$ have been obtained from the Arrhenius plot where it has been assumed that $\sigma \mathrm{s}$ are independent of temperature. Thus, taking $T$ as a constant in the above relation, the ratio of capture cross-sections can be written as $\sigma_{\perp} / \sigma_{\|}=\left(\varepsilon_{\|} / \varepsilon_{\perp}\right)^{2}$. The values of dielectric constants are $\varepsilon_{\|}=7.7$ and $\varepsilon_{\perp}=5.0$. Thus the ratio of capture cross-sections is found to be 2.4. It is found experimentally that $\sigma_{\perp}\left(\mathrm{H}_{3}\right)=5.0 \times 10^{-15} \mathrm{~cm}^{2}$ and $\sigma_{\|}\left(\mathrm{H}_{2}\right)=1.7 \times$ $10^{-15} \mathrm{~cm}^{2}$. Thus the ratio of measured capture cross-section $\left(\sigma_{\perp} / \sigma_{\|}\right)$is found to be 2.95 which agrees reasonably well with simple theory based on dielectric constant anisotropy. It was also observed that the energy levels of $\mathrm{H}_{2}$ and $\mathrm{H}_{3}$ are almost identical. Taking the above possible reasons for differences in $\sigma_{\infty}$ and $N_{1}$ into consideration, it may be concluded that $\mathrm{H}_{2}$ and $\mathrm{H}_{3}$ have the same physical origin.

The resistivity and Hall-effect results showed pronounced anisotropy in mobility (table 2). The activation energies found in two directions were also found to be different with $E_{a \|}=0.023 \mathrm{eV}$ and $E_{a \perp}=0.04 \mathrm{eV}$. These values, which can be understood on the basis of a modified hydrogenic model, involve the effective masses and dielectric constants which are anisotropic. Deep levels, on the other hand, arise due to strong potential perturbations and, according to some theories, are considered to be tied to the vacuum level (Langer and Heinrich 1985) or an internal bulk reference level (Tersoff and Harrison 1987). The independence of the deep level energy on the orientation of the semiconductor as found from the present experiment may be understood on this basis and provides indirect support to the latter theories. 
The present results may be compared with DLTS studies on p-GaSe which revealed trap energies at $0.34 \mathrm{eV}$ and $0.59 \mathrm{eV}$ above the valence band. Space charge limited current (SCLC) measurements on undoped p-GaSe (Manfredotti et al 1974) had shown the presence of deep hole traps at $0.421 \mathrm{eV}$ and $0.465 \mathrm{eV}$ above $E_{\mathrm{v}}$ with concentrations between $10^{12} \mathrm{~cm}^{-3}$ and $10^{13} \mathrm{~cm}^{-3}$. The capture cross-sections of these giant traps were estimated to be $\approx 10^{-12} \mathrm{~cm}^{2}$. Surprisingly the hole drift mobility perpendicular to the layer planes was found to be $\mu_{\perp}=215 \mathrm{~cm}^{2} \mathrm{~V}^{-1} \mathrm{sec}^{-1}$ larger than Hall mobility $\mu_{\|}=40 \mathrm{~cm}^{2} \mathrm{~V}^{-1} \mathrm{sec}^{-1}$, which is contrary to the present results on p-GaTe and on other layer type chalcogenides. In contrast DLTS on Sn-doped n-InSe showed only shallow levels with $E_{\mathrm{d}}=0.056 \mathrm{eV}$ and $0.074 \mathrm{eV}$ and one deep level $E_{\mathrm{c}}=0.11 \mathrm{eV}$ for crystals oriented parallel to the layer planes. These levels were attributed to Sn acting as donor.

Impurity analysis of GaTe sample by inductively coupled plasma (ICP) emission showed that the main impurities are $\mathrm{Pb}, \mathrm{Sn}, \mathrm{Al}, \mathrm{As}, \mathrm{Mg}, \mathrm{Zn}$ and $\mathrm{Fe}$, all below ppm level. $\mathrm{Pb}$ has been shown to be responsible for $\mathrm{p}$-type behaviour in InSe, $\mathrm{Sn}$ gives rise to n-type conduction and $\mathrm{Al}$ is iso-electronic with $\mathrm{Ga} . \mathrm{Mg}, \mathrm{Zn}$ and As are expected to be shallow acceptors at the Ga and Te sites respectively. Thus, the deep levels may be associated with Fe which is known to give rise to such levels in III-V and II-VI compounds or, as is quite likely, with unspecified native defects.

\section{Conclusion}

In this paper DLTS results on p-GaTe are presented utilizing Schottky barriers parallel and perpendicular to layer planes. In the parallel plane $\mathrm{H}_{3}(0.45 \mathrm{eV}), \mathrm{H}_{4}$ and an unresolved trap $\left(\mathrm{H}_{1}^{\prime}\right)$ corresponding to $\mathrm{H}_{1}(0.28 \mathrm{eV})$ were observed. Perpendicular to the layer plane also two traps $\mathrm{H}_{1}(0.28 \mathrm{eV})$ and $\mathrm{H}_{2}(0.42 \mathrm{eV})$ were found. $\left(\mathrm{H}_{2}, \mathrm{H}_{3}\right)$ appear to represent the same level with similar trap depth and capture cross-section. The trap $\mathrm{H}_{1}$ was also found in both directions. Thus, it may be concluded that while shallow acceptor levels in GaTe have orientation-dependent activation energies, the deep levels found have, within experimental error, energy levels which are independent of orientation.

\section{Acknowledgements}

The authors are grateful to the Department of Electronics, Government of India, for project support. We thank Dr Vikram Kumar, Director, Solid State Physics Laboratory, for help with fabrication of the DLTS system.

\section{References}

Bräunlich P, Kelly P and Fillara J -P 1979 in Thermally stimulated relaxation in solids (ed.) P Bräunlich (Berlin: Springer-Verlag) pp 50

Langer J M and Heinrich H 1985 Phys. Rev. Lett. 551414

Mari B, Segura A, Casanovas A and Chevy A 1991 Appl. Phys. A52 373

Martin P A, Hess K, Emanuel M and Coleman J J 1986 J. Appl. Phys. 602882

Manfredotti C, Murri R, Rizzo A, Galassini S and Ruggiero L 1974 Phys. Rev. B10 3387

Shigetomi S, Ikari T, Nakashima H and Nishimura H 1991 Phys. Status Solidi (a) 128 K95

Telia A, Lepley B and Michel C 1991 J. Appl. Phys. 697159

Tersoff J and Harrison W A 1987 Phys. Rev. Lett. 582367 